\title{
Introduction to Mobile Value Services Minitrack
}

\author{
Pirkko Walden \\ Institute for Advanced Management \\ Systems Research (IAMSR) Abo \\ Akademi University Turku, Finland \\ pirkko.walden@abo.fi
}

\author{
Tomi Dahlberg \\ University of Turku, School of \\ Economics Turku, Finland \\ tomi.dahlberg@utu.fi
}

\author{
Esko Penttinen \\ Aalto University, School of Business, \\ Helsinki, Finland \\ esko.penttinen@aalto.fi
}

At HICSS-35 in 2002 the first minitrack on Mobile Commerce was introduced. At that time mcommerce was an emerging field in its early stages with a number of ideas of what is going to constitute the key success factors for the actors in the global mcommerce arena. Back then we wrote 'It is plausible to assume that this area will be an important research topic for many years to come' - it still is after 15 years.

During the next three conferences the theme for the minitrack remained the same. We were in essence working on finding a conceptual framework for mcommerce products and services.

Four years later at HICSS-39 the theme for the minitrack was refocused as mobile value services. Mobile value services are designed as functional adaptations to the user context in such a way that they make user actions and activities more effective and/or more productive and/or working with less cost and/or possible to carry out with less use of resources and/or are able to simplify user routines in such a way that all the previous effects will be realized. The mobile value services designs (methods and models) aim at services which will change the limits of the possible in the structure of everyday life, which will contribute to making them everyday routines for the general population, i.e. services which will be used continually and as part of everyday activities.

From 2006 onwards the theme for the minitrack has been mobile value services or mobile value services plus some topical issue such as mobile business and mobile cloud at HICSS-46 in 2012.

Over the years enormous growth and development in mobile technologies as well as applications and services in the business world has taken place. At the same time our understanding of business models, platforms, eco-systems and value creation is not as advanced as necessary to contribute to sound dynamic modeling of the phenomena, to derive theoretical explanations or to provide guidance for these developments. To overcome this lack of understanding research contributions that open up new perspectives and offer insights for a better use of mobile technology has been the goal of the minitracks since the very start.
In this year's mini-track four papers are presented that offer a representative view on different aspects of mobile value services. The contributions were selected from 10 submitted papers after extensive peer reviews and one round of revisions:

In their paper Designing Effective Performance Feedback Notification Systems to Stimulate Content Contribution: Evidence from a Crowdsourcing Recipe Platform, by $\mathrm{Ni}$ Huang et al. a field experiment in China was conducted in order to study user content contribution which is of prime importance for many online platforms. Of particular interest is performance feedback, framing of feedback message and the relationship between gender and the framing of feedback message.

Second, Bonazzi et al. explore in their paper Healthy lottery. An economically viable mobile system to increase compliance of individuals with diabetes, a solution to improve self-management of older patients with type II diabetes using mobile technologies and incentives to guide and maintain long-term engagement.

The third paper My Wellness as a Mobile App. Identifying Wellness Types among the Young Elderly, by Sell et al. aims at identifying segments within the young elderly age group. The "ageing population" is a broad and ill-defined segment of the population, whereby improved understanding of how to conceptualize segments within it is offered. Preliminary results of a mixed-method study of young elderly and their use of mobile applications are presented.

The fourth paper Assimilation of Mobile Marketing in Organisations, by Stanoevska-Slabeva et al. addresses the implementation and assimilation of various mobile marketing technologies from a company point of view. Applying a mixed-method approach some interesting results were obtained; culture has a significant impact on how mobile marketing goals are achieved and three segments could be identified. 